\title{
Percutaneous Stenoscopic Lumbar Decompression with Paramedian Approach for Foraminal/ Extraforaminal Lesions
}

\author{
Han Ga Wi Nam, Hyung Suk Kim, Dong Keun Lee, Chun-Kun Park, Kang Taek Lim \\ Department of Neurosurgery, Good Doctor Teun Teun Hospital, Anyang, Korea
}

The lumbar foramen is affected by different degenerative diseases, including extraforaminal disc herniation, foraminal stenosis (FS), and degenerative or spondylolytic spondylolisthesis. The purpose of this study was to describe percutaneous stenoscopic lumbar decompression with a paramedian approach (para-PSLD) for foraminal/extraforaminal lesions. All operative procedures were performed using a complete uniportal endoscopic instrument system. The para-PSLD can be easily applied to patients with FS and narrow disc space or facet joint hypertrophy. The anatomical view of a para-PSLD is similar to that of a conventional open surgery and allows for good visualization of the foraminal/extraforaminal areas. We suggest that para-PSLD is an alternative and minimally invasive procedure to treat degenerative lumbar foraminal/extraforaminal stenoses.

Keywords: Spinal stenosis; Foraminal stenosis; Percutaneous stenoscopic lumbar decompression; Endoscopic spinal surgery; Paramedian approach

\section{Introduction}

Degenerative lumbar foraminal stenosis (FS) is a relatively common cause of lumbar radiculopathy, with a reported incidence of $8 \%-11 \%$ [1-4]. The lumbar foramen is affected by different degenerative diseases, including extraforaminal disc herniation, FS, and degenerative or spondylolytic spondylolisthesis such as far-out syndrome $[1,5,6]$. Various surgical procedures have been used to treat these lesions, ranging from microscopic decompression via various approaches to fusion with or without total facetectomy [1-3,7-11]. Conventional microscopic decompressions for foraminal/extraforaminal lesions through the paramedian approach require large skin incisions and extensive muscle retraction [12]. Microscopic decompression using a tubular retractor has the advantage of preserving the back muscles $[9,13]$. However, the handling of various instruments through a long and narrow retractor, with the added challenge of poor visibility, makes the procedure difficult [12]. Traditional transforaminal endoscopic surgery is performed via Kambin's triangle, which is often very narrow in foraminal and extraforaminal stenoses $[14,15]$. Moreover, traditional transforaminal endoscopic spine surgery docked into Kambin's triangle cannot easily eliminate bony lesions under stenotic conditions and increases the risk of neural irritation and damage [16]. To overcome these hurdles, we introduce a modified full-endoscopic procedure using a stenoscope (a $12^{\circ}$ endoscope) through a paramedian surgical approach to treat foraminal and extraforaminal stenoses with or without disc

Received Oct 14, 2018; Accepted Nov 20, 2018

Corresponding author: Kang Taek Lim

Department of Neurosurgery, Good Doctor Teun Teun Hospital, 775 Gyeongsu-daero Dongan-gu, Anyang 14041, Korea

Tel: +82-31-8086-8357, Fax: +82-2-534-4799, E-mail: limkat@hanmail.net 
herniation. Accordingly, the purpose of this study was to describe percutaneous stenoscopic lumbar decompression with a paramedian approach (para-PSLD) for foraminal/ extraforaminal lesions.

\section{Technical Note}

\section{Indications}

Three spine surgeons performed 85 lumbar foraminal/extraforaminal stenosis decompressions with para-PSLD between May 2017 and May 2018. Our inclusion criteria were (1) unilateral radiculopathy correlated with foraminal/ extraforaminal disc herniation or stenosis and (2) failed conservative 6-week treatment. The exclusion criteria included the following: (1) bilateral radiculopathy and (2) instability defined as an angular motion translation of $4 \mathrm{~mm}$ or $10^{\circ}$. We observed 54 patients with FS and collapsed disc heights, 26 patients with FS and degenerative spondylolisthesis, 10 patients with FS and adjacent segment disease after fusion surgery, three patients with FS and spondylolytic spondylolisthesis, eight patients with extraforaminal lumbar disc herniation, and five patients with far-out syndrome and spurs from the L5 transverse process. In total, the procedures were performed using a left-side approach in 61 cases and a right-side approach in 45 cases.

\section{Anesthetic technique}

Careful monitoring by an anesthesiologist is critical during the operation because the patient is not under general anesthesia and is undergoing surgery in the prone position. Epidural anesthesia is performed one or two levels above the index level. Conscious sedation with sevoflurane $(1-2 \mathrm{vol} / \%)$ is allowed. This method is useful for patients who cannot undergo general anesthesia and reduces the risk of side effects such as reduced nausea, dysphagia, and memory loss.

\section{Approach and general technique}

We performed the procedure with the patient lying comfortably in the prone position on a radiolucent table (Fig. 1A). All operative procedures were performed using a complete uniportal endoscopic instrument system, the $\mathrm{H}$ View Endoscopic System (H-View, Daejeon, Korea) (Fig. 1B). We used a bipolar radiofrequency electro-coagulator
(OK Medinet Korea, Seoul, Korea) (Fig. 1C) for hemostasis, clearance of soft tissue, annulotomy, and annuloplasty. We used the same conventional surgical instruments during the endoscopic surgery by modifying their working length (Fig. 2). During surgery, we placed the fluoroscope on the operating table. Under fluoroscopic guidance, the surgeon made a $7-\mathrm{mm}$ incision $4-5 \mathrm{~cm}$ lateral to the spinous processes (the incision point may vary depending on the lesion location), central to the intervertebral foramen to be decompressed, exposing the lumbar foraminal or extraforaminal zone via a paramedian approach. The main target of this procedure is not the herniated disc fragment but the foraminal nerve root entrapped by bony stenoses and thickened foraminal ligaments. Thus, the surgeon advanced a blunt dilator with a $9.5-\mathrm{mm}$ outer diameter as a guide for the working sleeve into the lateral margin of the ipsilateral side isthmus (Fig. 3). Unlike the transforaminal endoscopic techniques, the para-PSLD approach does not directly cross over the exiting root. Therefore, the core of this technique is the docking to the isthmus (Fig. 4). Next, the surgeon inserted the working sleeve over the dilator
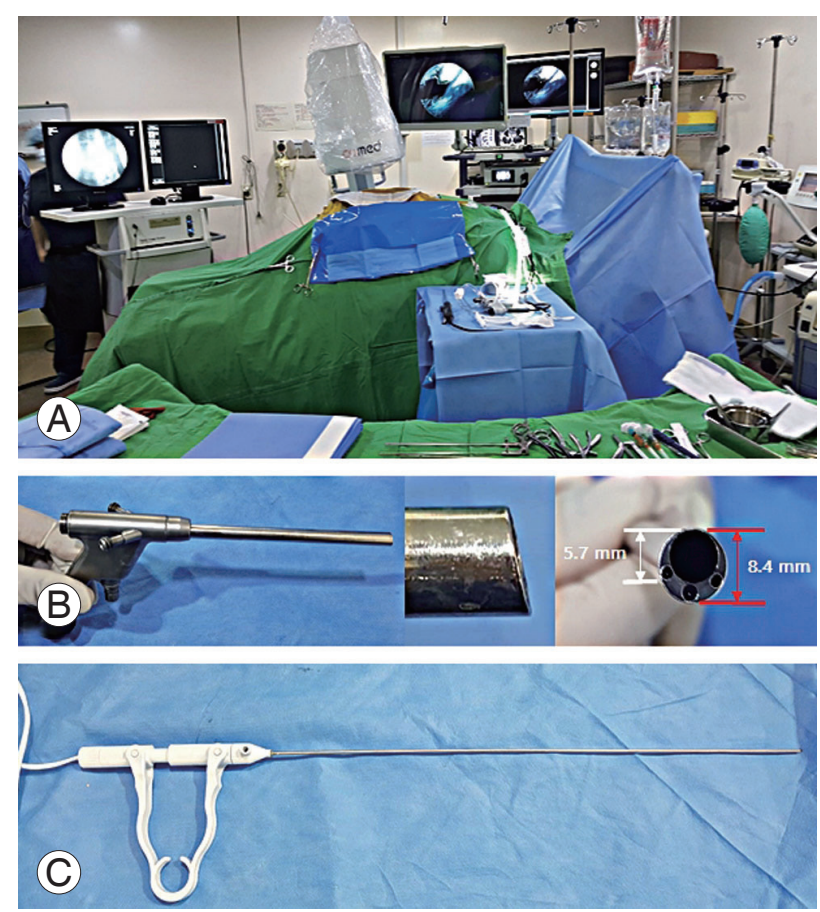

Fig. 1. (A) Preparations for percutaneous stenoscopic lumbar decompression with a paramedian approach operation. The procedure was performed with the patient comfortably lying in the prone position on a radiolucent table. (B) A rigid angle stenoscope, a type of endoscope with an 8.4-mm outer diameter, 5.7-mm working diameter channel, and a $12^{\circ}$ view. (C) Bipolar radiofrequency electro-coagulator. 
and introduced a rigid angle stenoscope (a type of endoscope), with an 8.4-mm outer diameter and $12^{\circ}$ view into the lesion from one side. The surgeon maintained direct endoscopic visual control with constant irrigation with an $\mathrm{H}$-View Endoscopic System through the following sequence of steps (Fig. 5, Supplemental Video 1). (1) The superolateral part of the caudal level superior articular process (SAP) is resected using Kerrison punches and a high-speed drill. (2) The lateral isthmus portion and superomedial SAP are resected with the endoscope tilted toward the medial side. This procedure creates space for the stenoscope to work; it is critical to have enough space for the endoscope in patients with narrowed disc space hypertrophy of SAP. (3) After confirming the dissection of the upper pedicle and excision of the intertransverse and foraminal ligaments, the affected exiting nerve root is exposed; adequately decompressing the L5 nerve root from the medial part of the superior facet to the sacral ala is critical. Osteophytes of the vertebral body are removed using a high-speed drill, and partial L5 or S1 pedicles are
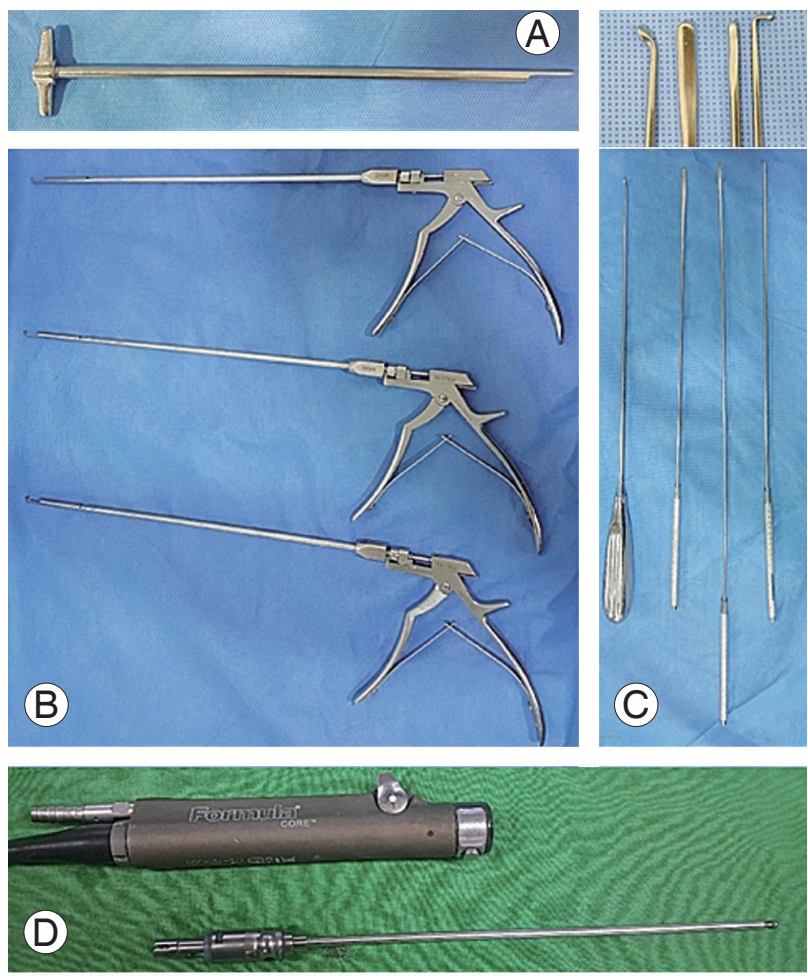

Fig. 2. Preparations for percutaneous stenoscopic lumbar decompression with a paramedian approach operation instrument set. (A) Specialized root retractor for endoscopic spinal surgery. (B) Kerrison Rongeurs in 2, 3, and $4 \mathrm{~mm}$ (available up to 5-mm size) shown from upper-to-lower. (C) Long curette, large dissector, small dissector, and ball-tipped probe shown from left to right. (D) The high-speed drill. removed as needed. (4) Next, the radicular artery is located and coagulated to control the bleeding. (5) Kerrison punches $(2-4 \mathrm{~mm})$ are used to decompress the medial side of the lateral edge of the yellow ligament. (6) The nerve structures are then freed with dissectors and freers. (7) If the patient has extraforaminal stenoses, additional extraforaminal decompressions are performed by tilting the stenoscope to the lateral side.
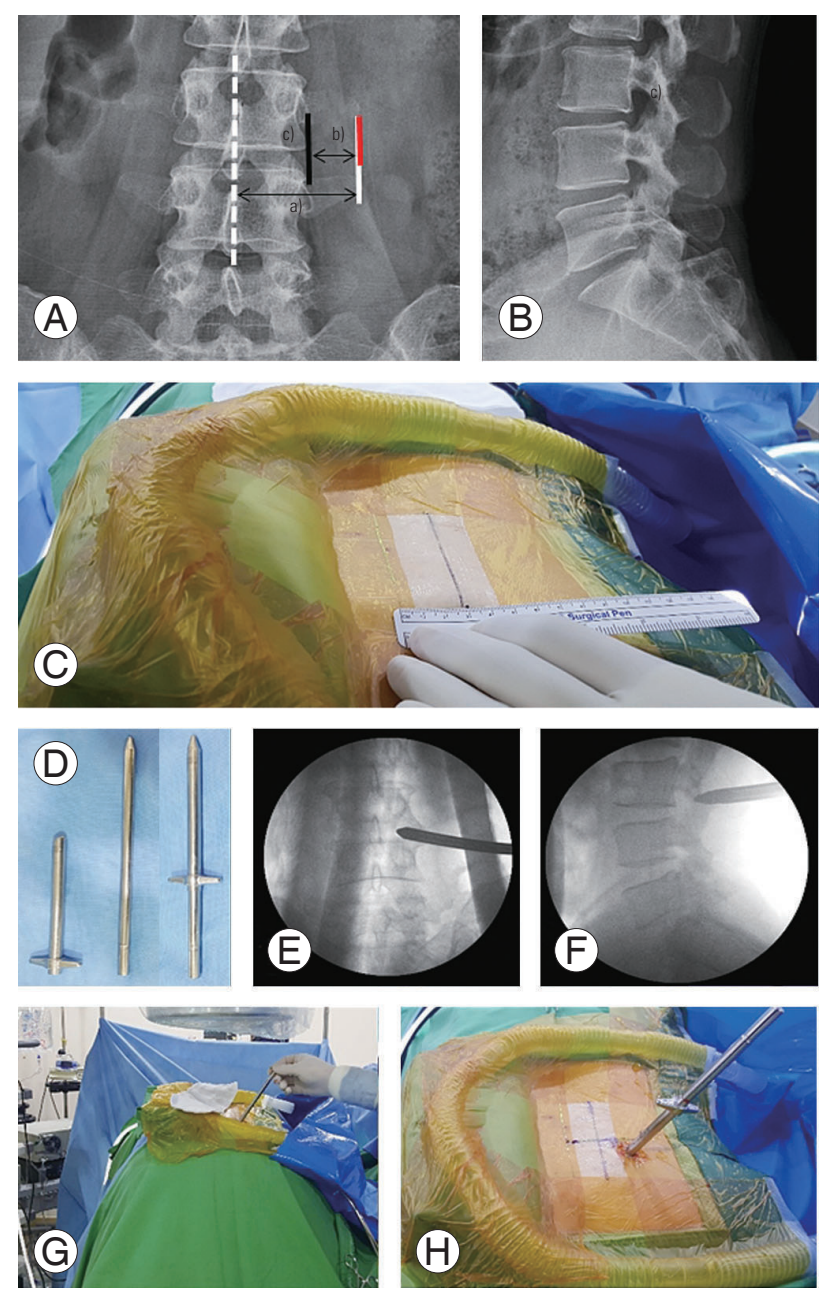

Fig. 3. Operative procedure (from initial skin incision to working cannula insertion). (A, B) Skin incision and target point for obturator docking. The white dashed line indicates the midline, the black line indicates the lateral border of the pedicle line, and the red line indicates a

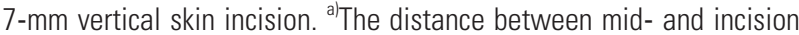
lines $(4-6 \mathrm{~cm})$; b) the distance between the lateral pedicle line border and incision line $(1-1.5 \mathrm{~cm})$; and ${ }^{\mathrm{c}}$ target point for initial docking. (C) Photograph showing the midline and the pedicle line lateral border at the surgical site. (D) Photograph of an obturator, working sleeve, and two instruments assembled prior to insertion into the surgical site. ( $\mathbf{E}_{\text {, }}$ F) Anteroposterior and lateral fluoroscopic views of the docked obturator on the isthmus. (G, H) Final position of the obturator at the surgical site. 

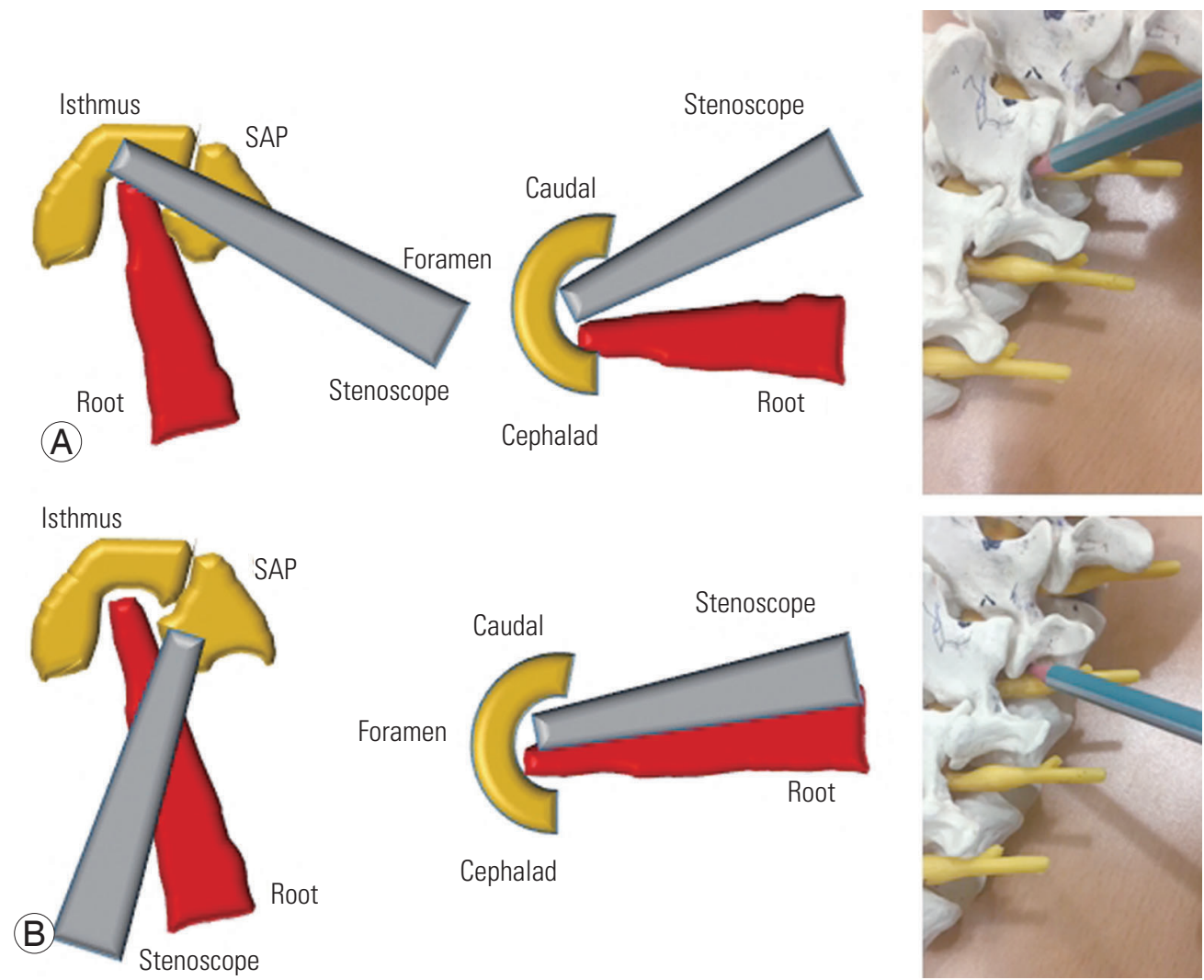

Cephalad

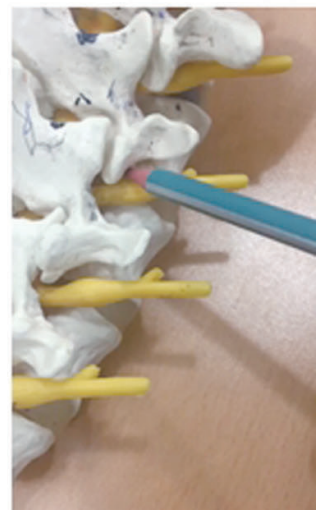

Fig. 4. Schematic comparing para-PSLD with a transforaminal approach. The transforaminal approach is likely to cause injury to the exiting root because the endoscope and root overlap with each other. Para-PSLD is not directed at Kambin's triangle, so it is unlikely to injure the exiting root. (A) Para-PSLD. (B) Transforaminal approach. Para-PSLD, preparations for percutaneous stenoscopic lumbar decompression with a paramedian approach; SAP, superior articular process.

\section{Operative orientation and advanced technique}

After inserting a scope over the intertransverse space, the bony margins, rather than the foramen itself, should be secured for orientation. If it is difficult to determine the exact location of the structure, positioning should be rechecked using the fluoroscope. The foramen entrance (medial), under-surface of the SAP (dorsal), and intertransverse ligament (basal) can be differentiated with correct orientation. Special care should be taken to not injure the radicular artery or proceed ventrally farther than the intertransverse ligament where the psoas muscle is. We resected the hypertrophied tip of the SAP using a highspeed drill and a Kerrison punch. Then, we detached the proximal margin of the foraminal ligament flavum from the distal surface of the transverse process and cranial level pedicle. Care should be taken to not injure the radicular artery and the exiting root just beneath the distal margin of the transverse process at the cranial nerve level; the artery may be hidden under the transverse process and beneath a degenerative, fibrous vascular bundle which can be mistaken for a thin atrophic root. We palpated the disc space after retraction of the exiting nerve root. Then, we proceeded to remove disc herniations that were seen to indent, compress, or displace the nerve root, and if needed, we performed intradiscal nucleotomies on a caseby-case basis. We performed discectomies when needed, after locating the root margin and controlling any bleeding from small vessels overlying the root. Then we used a bipolar radiofrequency electro-coagulator to perform the annulotomy and inserted the pituitary forceps and Kerrison punches just beneath the outer layer of the annulus to remove ruptured disc fragments, particularly those located under the root and far laterally at the crossing point between the root and the annulus. Finally, we applied postoperative drainage and removed the drain one day post operation. Postoperative magnetic resonance imaging (MRI) revealed adequate decompression in all cases. 

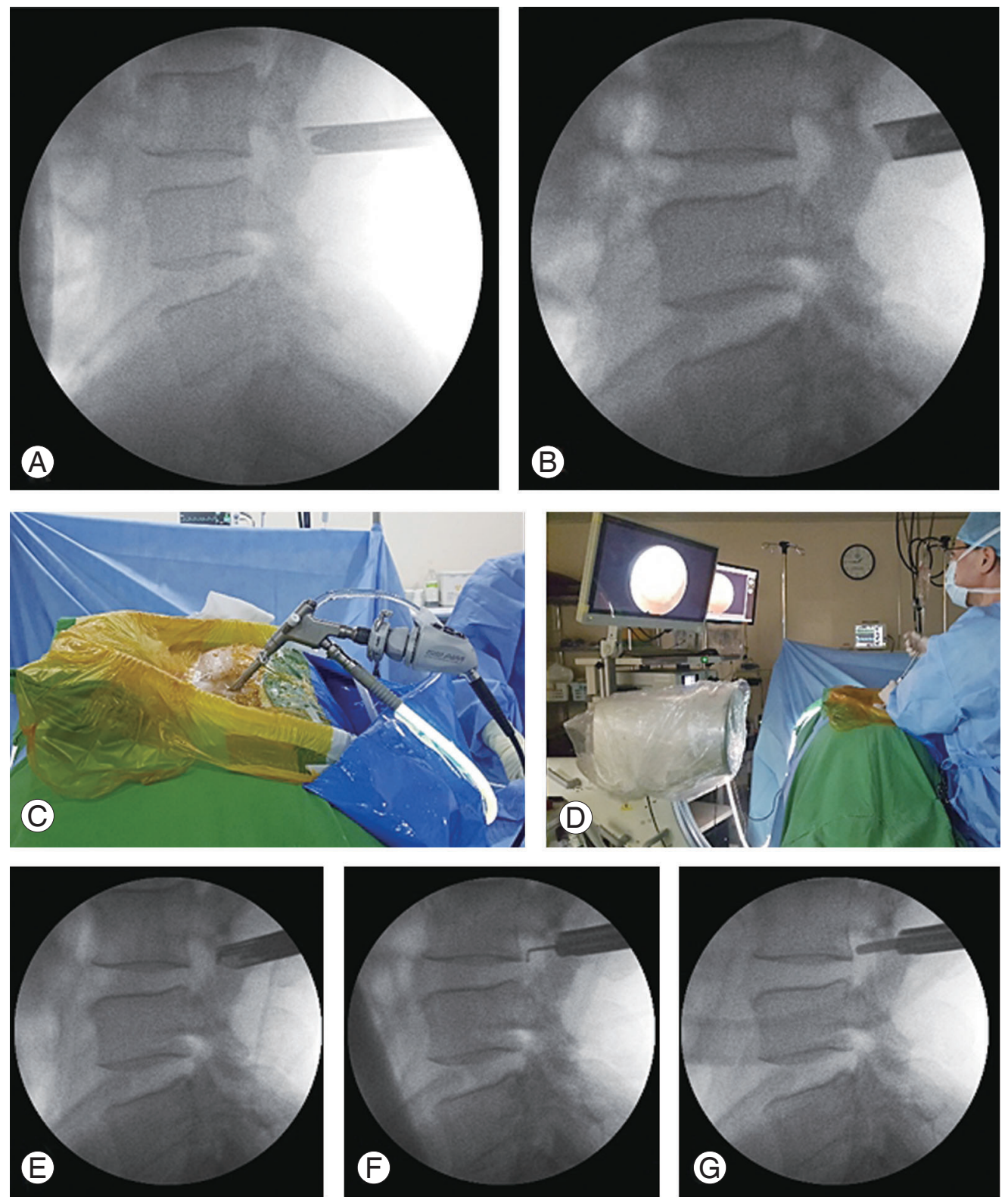

Fig. 5. Operative procedure of foraminal decompression. (A) Final position of the obturator and the working sleeve on the surgical site (isthmus). (B) Stenoscope insertion through the working sleeve. (C, D) The procedure was performed under direct endoscopic visual control with constant irrigation and by tilting the endoscope toward the foramen. (E-G) Foramen confirmation and foraminal decompression using Kerrison Rongeurs, a ball-tipped probe, and dissectors.

Representative cases are illustrated in Figs. 6-9.

\section{Discussion}

Endoscopic operations, such as arthroscopy and laparoscopy, have become clinical standards [17]. Lumbar spinal diseases range from simple disc herniations to complicated cases such as highly migrated disc herniations and other pathologies combined with bony degeneration producing foraminal and canal stenoses. These cases can also be treated with full-endoscopic operations using various methods of access and techniques [18-24]. Spinal endoscopy allows the surgeon to expeditiously reach a deep site without causing excessive bleeding or muscle damage and thus provides a high-quality magnified visual field. Para-PSLD can be performed with just one portal to access spinal pa- 

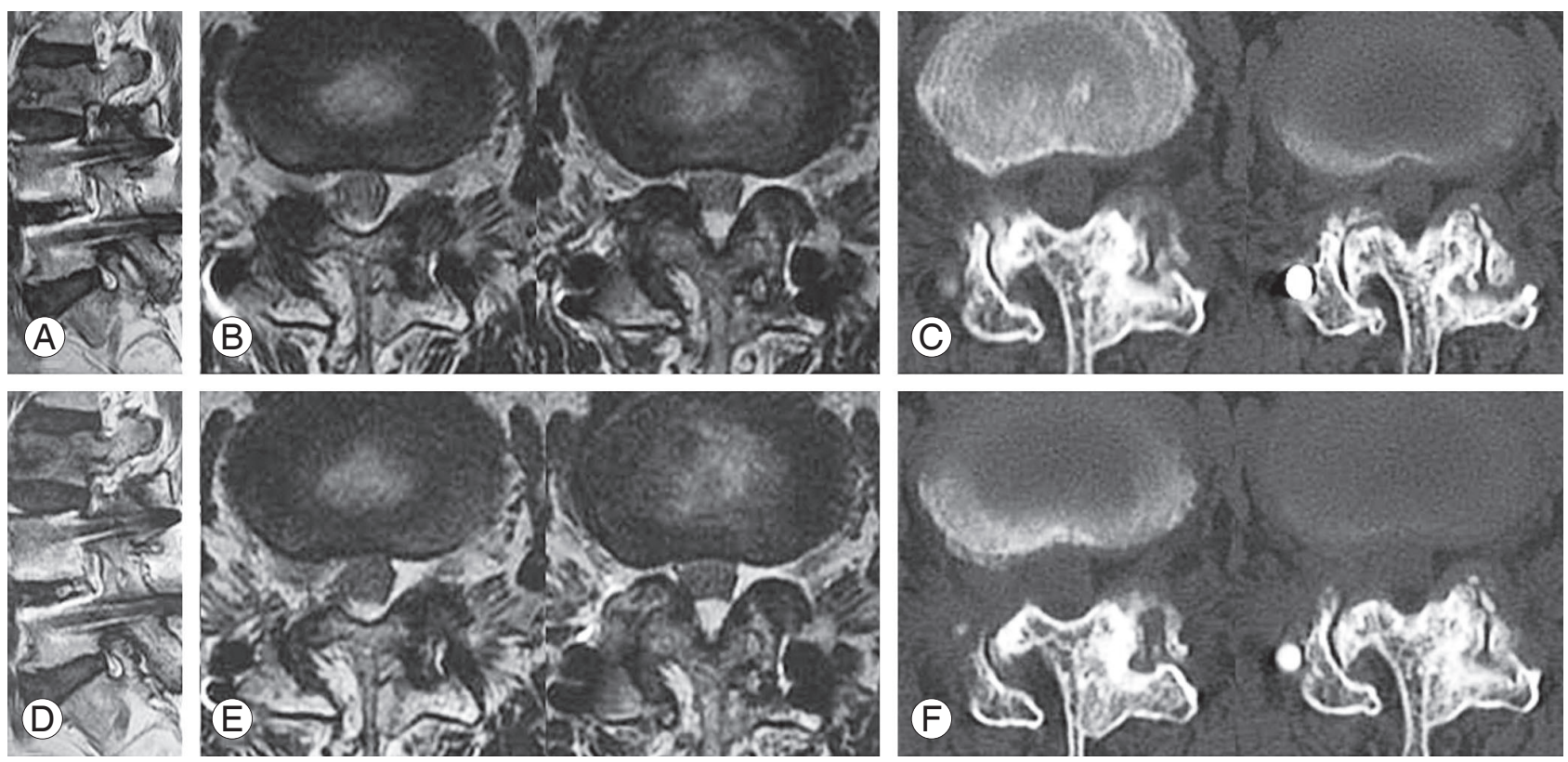

Fig. 6. Preparations for percutaneous stenoscopic lumbar decompression with a paramedian approach in a patient with right L3-L4 foraminal stenosis and $\mathrm{L} 3$ acute compression fracture (the patient underwent interbody fusion of $L 4-L 5$ with pedicle screw and rod fixation). (A, B) Preoperative sagittal and axial MRI. (C) Preoperative axial CT images. (D, E) Postoperative sagittal and axial MRI. (F) Postoperative axial CT images. MRI, magnetic resonance imaging; CT, computed tomography.
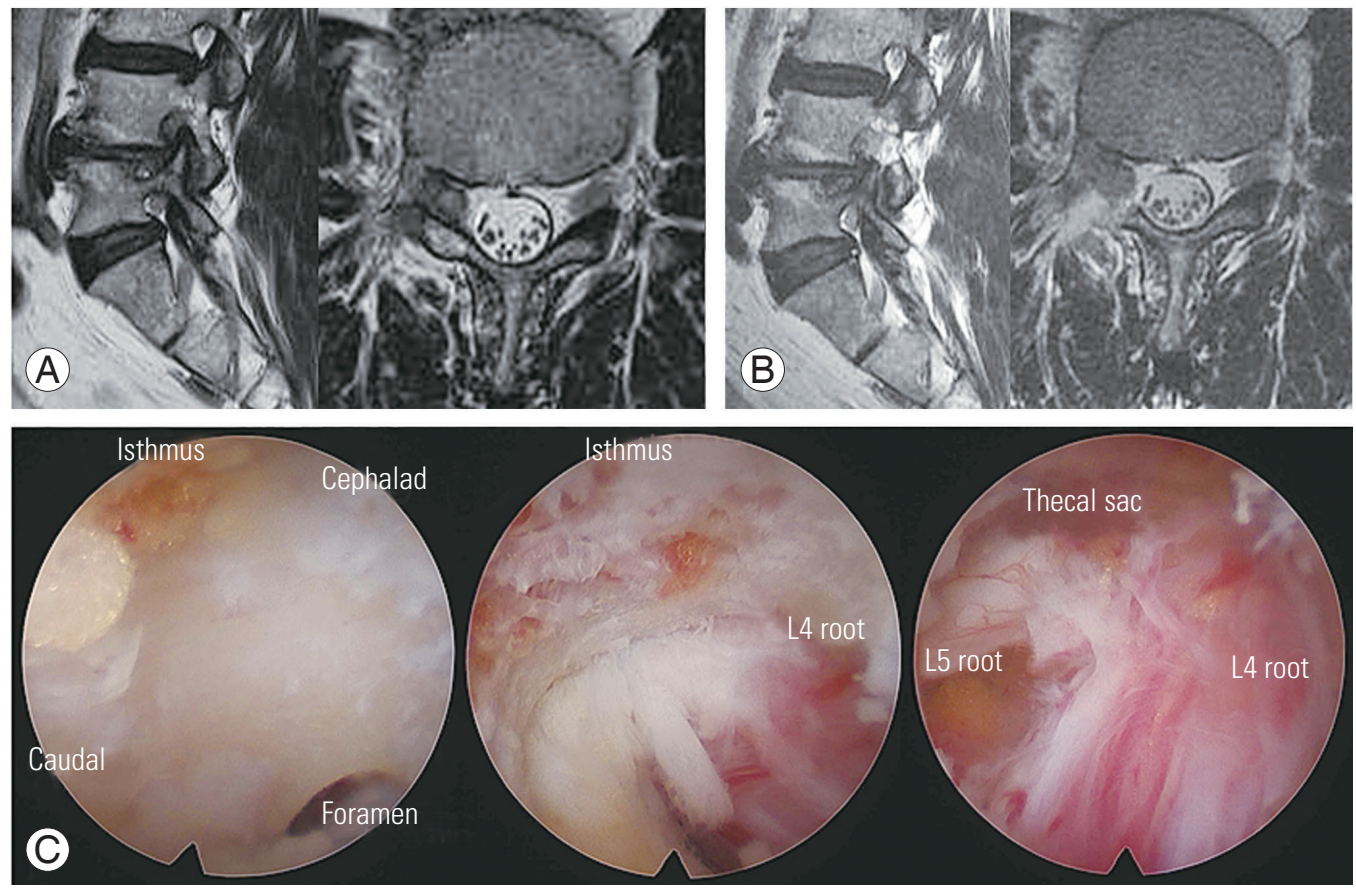

Fig. 7. Preparations for percutaneous stenoscopic lumbar decompression with a paramedian approach in a patient with right L4-L5 foraminal stenosis. (A) Preoperative sagittal and axial MRI. (B) Postoperative sagittal and axial MRI. (C) Intraoperative endoscopic images. MRI, magnetic resonance imaging.

thologies and differ from percutaneous transforaminal endoscopic lumbar discectomy and decompression via Kambin's triangle. Traditional transforaminal endoscopic spine surgery docked into the Kambin's triangle cannot easily eliminate bony lesions in stenotic conditions because of the increased risk of neural irritation and damage. In contrast, 



Fig. 8. Preparations for percutaneous stenoscopic lumbar decompression with a paramedian approach in a patient with left $L 4-\mathrm{L} 5$ foraminal stenosis. (A, B) Preoperative sagittal and axial MRI. (C) Preoperative axial CT images. (D, E) Postoperative sagittal and axial MRI. (F) Postoperative axial CT images (the white arrow indicates the location of the drainage catheter). MRI, magnetic resonance imaging; CT, computed tomography.
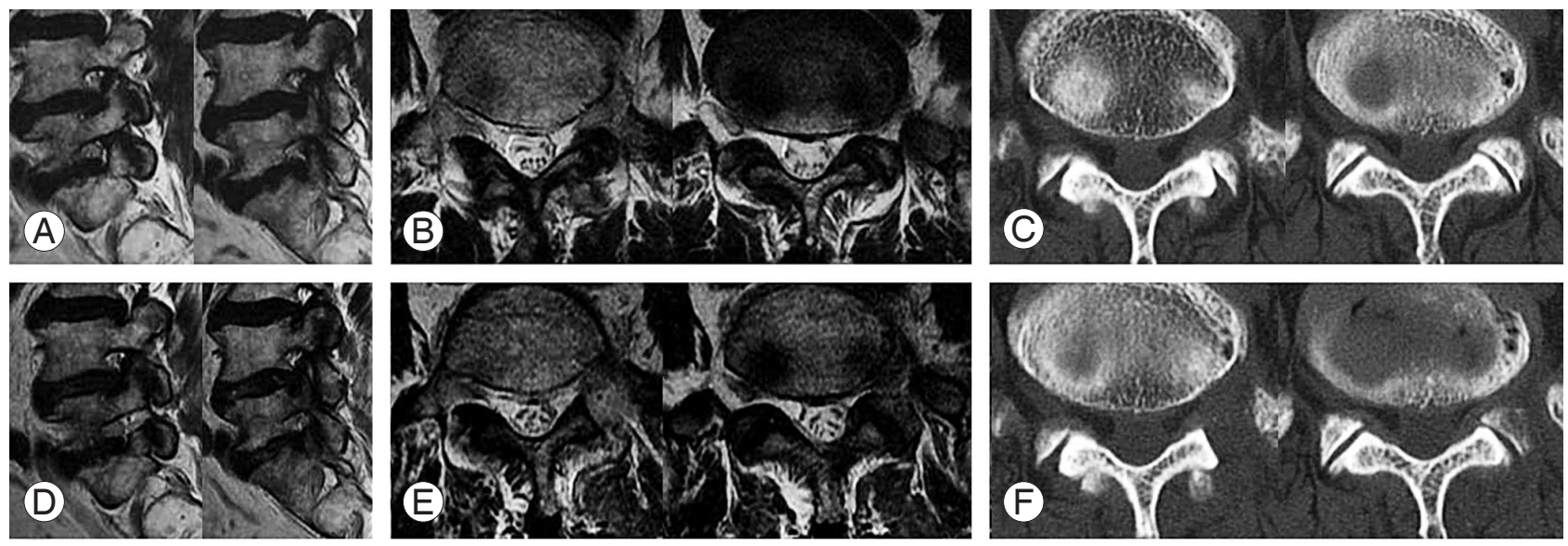

Fig. 9. Preparations for percutaneous stenoscopic lumbar decompression with a paramedian approach in a patient with left L5-S1 foraminal stenosis. (A, B) Preoperative sagittal and axial MRI. (C) Preoperative axial CT images. (D, E) Postoperative sagittal and axial MRI. (F) Postoperative axial CT images. MRI, magnetic resonance imaging; CT, computed tomography.

para-PSLD can access the lesion after directly confirming the location of the exiting root, and thus, the operation can be performed with minimal injury to the root. In addition, para-PSLD can be easily applied to patients with FS and narrow disc space or facet joint hypertrophy as access to Kambin's triangle is no longer needed.

We designated para-PSLD as our procedure of choice for most patients with stand-alone foraminal/extraforaminal stenoses. With para-PSLD, we were able to approach and easily decompress the nerve root and dorsal root ganglion (DRG) residing in the foramen. This approach provided excellent surgical views for good decompres- sion of DRG under direct vision. As a result of the lateral to medial viewing angle, we decompressed the exit and midzone of the foramen without sacrificing too much of the pars interarticularis or the facet joint. We confirmed the minimal removal of the facet joint in the computed tomography images in Figs. 6-9. The procedure is minimally invasive, and the only structures that are required to be sacrificed are the medial intertransverse muscle, the tip of SAP, and the lateral portion of the pars interarticularis. With extensive use of the high-speed drill, resection of a large portion of the facet joint is not needed. Therefore, this approach carries little risk of postoperative instability. 


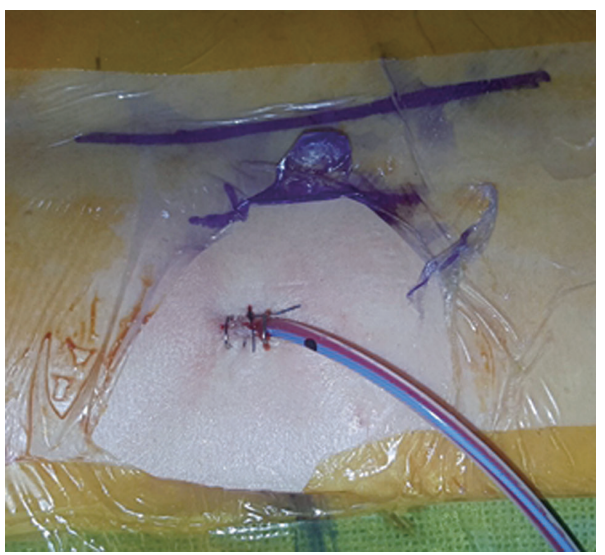

Fig. 10. Photograph showing skin incision and drainage sites. The operation can be performed through a skin incision of only 7-8 $\mathrm{mm}$, and the drainage tube can be inserted through the same incision site.
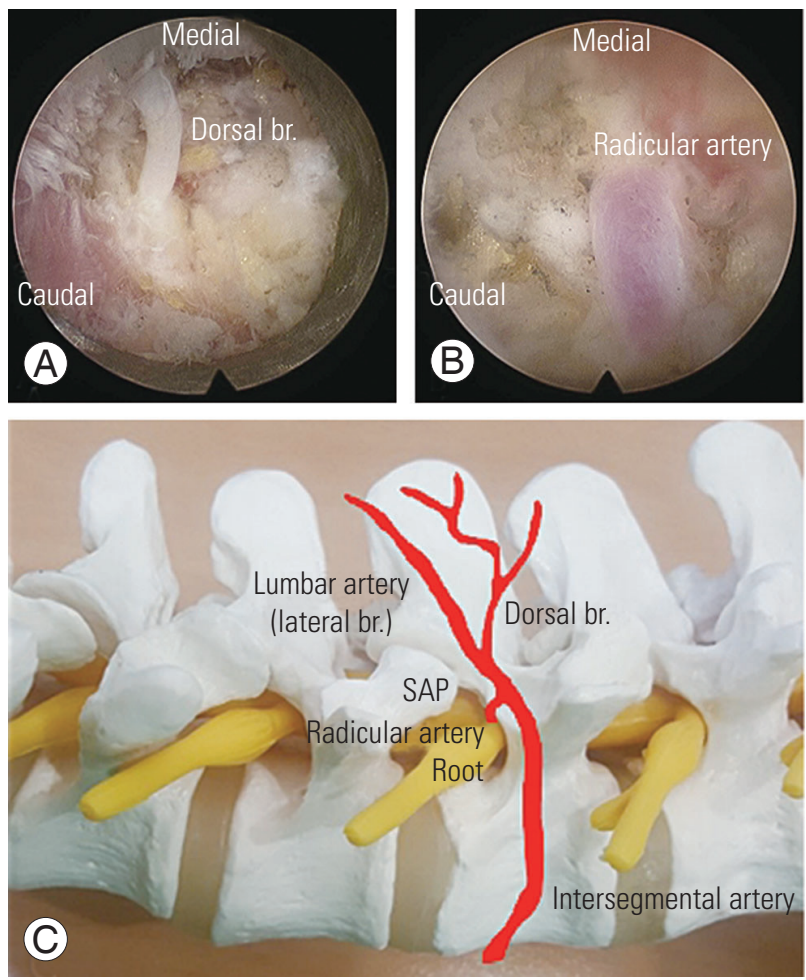

Fig. 11. Foraminal structures. (A, B) Intraoperative endoscopic images. (C) Schematic anatomy of the foraminal structures. During the removal of the intertransverse ligament, the dorsal branch artery is located first. A closer foramen approach can reveal the location of the radicular artery. br, branch; SAP, superior articular process.

Additionally, it is possible to perform the operation with a single skin incision of 7-8 $\mathrm{mm}$ in size, minimizing tissue damage and maximizing the cosmetic effect (Fig. 10).

There are several technical pitfalls that warrant discussion. Technically, placing the working tube in the lateral margin of the isthmus is the most important procedure
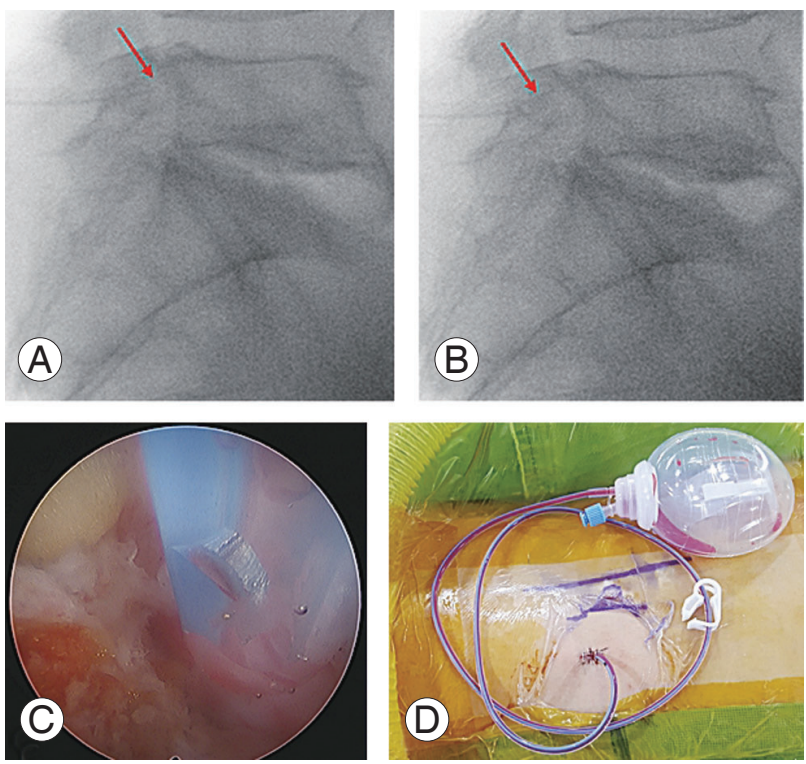

Fig. 12. (A) Intraoperative fluoroscopic image showing the drainage tip inserted too deeply (the red arrows indicate the location of the drainage tips). (B) Intraoperative fluoroscopic image showing the drainage tip inserted in the proper position. (C) Photograph showing endoscopic-guided drain insertion. (D) Photograph showing skin suture and final drainage insertion.

of para-PSLD and must be performed with great care. The positions of the lateral margins of the facet joints are determined through preoperative radiological imaging; an imaginary line extending from these two positions determines the position of the incision on the midline, and the working tube must be placed toward the lateral border of the isthmus at the incision line (Fig. 3A). Furthermore, the tip of the ascending facet up to its most medial portion adjacent to the spinal canal must be resected to provide complete decompression of DRG. Failure to remove the most medial portion of SAP can lead to residual or recurrent symptoms.

A working tube is docked at the lateral border of the isthmus. At this time, under the stenoscope, the muscular attachments are freed from the transverse processes, and the intertransverse ligament is incised. The exiting nerve root is then identified and mobilized for removal of the underlying pathologies. The radicular artery is identified around the exiting root (Fig. 11). Taking great care of the radicular artery is important to keep a clear surgical field and avoid root injury. A blurred surgical field due to radicular artery bleeding frequently interrupted our procedures. On occasion, we had to discontinue the operation due to extensive radicular artery bleeding. We controlled vascular bleeding using a bipolar radiofrequency electro- 
coagulator with little muscle retraction to pull the vessel backward at the time of current firing.

After the operation, a drain is inserted to prevent hematoma at the surgical site. Interestingly, immediately after the surgery, patients experienced severe pain similar to that before the procedure. Although most had no problems during the operation, patients complained of severe pain when lying on the bed and moving their legs or changing posture. We found that the drain tip, which was inserted at the surgical site during the operation, was stimulating the exiting root; moving the drain tip by just $5 \mathrm{~mm}$ improved the symptoms in most cases. To prevent this issue, we tested endoscopic-guided drain insertions or confirmed the location of the tip using a C-arm in the operative field (Fig. 12).

In our clinic, we performed nerve root blocks to establish diagnoses in all patients prior to surgery because MRI findings were either too subtle or were composed of multiple lesions, which made the determination of the offending lesion difficult. We performed the procedures under fluoroscopic control according to the technique previously described by Pfirrmann et al. [25]. In cases in which the procedure completely resolved the patient's symptoms (even transiently), we confirmed that the blocked nerve root was responsible for the symptom and considered those patients as good candidates for this operation. This block for diagnosis confirmation is likely important to ensure proper patient selection and satisfactory postoperative results.

In our study, we were only able to assess and follow up 106 patients. Therefore, our results cannot be easily extrapolated to other populations. To address this, a larger number of patients should be studied and followed up.

The anatomical view of para-PSLD is similar to that of a conventional open surgery and allows for good visualization of foraminal/extraforaminal areas. We suggest paraPSLD as an alternative minimally invasive procedure for the treatment of degenerative lumbar foraminal/extraforaminal stenoses.

\section{Conflict of Interest}

No potential conflict of interest relevant to this article was reported.

\section{Supplementary Materials}

Supplemental Video 1. PSLD paramedian approach L4-5 right. Data file can be available from https://doi. org/10.31616/asj.2018.0269.v001.

\section{References}

1. Jenis LG, An HS, Gordin R. Foraminal stenosis of the lumbar spine: a review of 65 surgical cases. Am J Orthop (Belle Mead NJ) 2001;30:205-11.

2. Kunogi J, Hasue M. Diagnosis and operative treatment of intraforaminal and extraforaminal nerve root compression. Spine (Phila Pa 1976) 1991;16:1312-20.

3. Porter RW, Hibbert C, Evans C. The natural history of root entrapment syndrome. Spine (Phila Pa 1976) 1984;9:418-21.

4. Weiner BK, Fraser RD. Foraminal injection for lateral lumbar disc herniation. J Bone Joint Surg Br 1997;79:804-7.

5. Matsumoto M, Watanabe K, Ishii K, et al. Posterior decompression surgery for extraforaminal entrapment of the fifth lumbar spinal nerve at the lumbosacral junction. J Neurosurg Spine 2010;12:72-81.

6. Epstein NE, Epstein JA, Carras R, Hyman RA. Far lateral lumbar disc herniations and associated structural abnormalities: an evaluation in 60 patients of the comparative value of CT, MRI, and myelo-CT in diagnosis and management. Spine (Phila Pa 1976) 1990;15:534-9.

7. Baba H, Uchida K, Maezawa Y, Furusawa N, Okumura Y, Imura S. Microsurgical nerve root canal widening without fusion for lumbosacral intervertebral foraminal stenosis: technical notes and early results. Spinal Cord 1996;34:644-50.

8. Chang SB, Lee SH, Ahn Y, Kim JM. Risk factor for unsatisfactory outcome after lumbar foraminal and far lateral microdecompression. Spine (Phila Pa 1976) 2006;31:1163-7.

9. Hallett A, Huntley JS, Gibson JN. Foraminal stenosis and single-level degenerative disc disease: a randomized controlled trial comparing decompression with decompression and instrumented fusion. Spine (Phila Pa 1976) 2007;32:1375-80.

10. Jenis LG, An HS. Spine update. Lumbar foraminal stenosis. Spine (Phila Pa 1976) 2000;25:389-94.

11. Ozeki N, Aota Y, Uesugi M, et al. Clinical results of 
intrapedicular partial pediculectomy for lumbar foraminal stenosis. J Spinal Disord Tech 2008;21:324-7.

12. Choi DJ, Kim JE, Jung JT, et al. Biportal endoscopic spine surgery for various foraminal lesions at the lumbosacral lesion. Asian Spine J 2018;12:569-73.

13. Epstein NE. Foraminal and far lateral lumbar disc herniations: surgical alternatives and outcome measures. Spinal Cord 2002;40:491-500.

14. Ahn Y, Lee SH, Lee JH, Kim JU, Liu WC. Transforaminal percutaneous endoscopic lumbar discectomy for upper lumbar disc herniation: clinical outcome, prognostic factors, and technical consideration. Acta Neurochir (Wien) 2009;151:199-206.

15. Ahn Y, Oh HK, Kim H, Lee SH, Lee HN. Percutaneous endoscopic lumbar foraminotomy: an advanced surgical technique and clinical outcomes. Neurosurgery 2014;75:124-33.

16. Wang YP, Zhang W, Li BL, Sun YP, Ding WY, Shen Y. Suprapedicular foraminal endoscopic approach to lumbar lateral recess decompression surgery to treat degenerative lumbar spinal stenosis. Med Sci Monit 2016;22:4604-11.

17. Lee CW, Yoon KJ, Jun JH. Percutaneous endoscopic laminotomy with flavectomy by uniportal, unilateral approach for the lumbar canal or lateral recess stenosis. World Neurosurg 2018;113:e129-37.

18. Lee CW, Yoon KJ, Ha SS, Kang JK. Foraminoplastic superior vertebral notch approach with reamers in percutaneous endoscopic lumbar discectomy: technical note and clinical outcome in limited indications of percutaneous endoscopic lumbar discectomy. J Korean Neurosurg Soc 2016;59:172-81.

19. Lee SH, Choi KC, Baek OK, Kim HJ, Yoo SH. Percu- taneous endoscopic intra-annular subligamentous herniotomy for large central disc herniation: a technical case report. Spine (Phila Pa 1976) 2014;39:E4739.

20. Ahn Y, Lee SH, Park WM, Lee HY. Posterolateral percutaneous endoscopic lumbar foraminotomy for L5-S1 foraminal or lateral exit zone stenosis. Technical note. J Neurosurg 2003;99(3 Suppl):320-3.

21. Sairyo K, Higashino K, Yamashita K, et al. A new concept of transforaminal ventral facetectomy including simultaneous decompression of foraminal and lateral recess stenosis: technical considerations in a fresh cadaver model and a literature review. J Med Invest 2017;64:1-6.

22. Telfeian AE, Jasper GP, Francisco GM. Transforaminal endoscopic treatment of lumbar radiculopathy after instrumented lumbar spine fusion. Pain Physician 2015;18:179-84.

23. Kim CH, Chung CK. Endoscopic interlaminar lumbar discectomy with splitting of the ligament flavum under visual control. J Spinal Disord Tech 2012;25:210-7.

24. Choi G, Lee SH, Lokhande P, et al. Percutaneous endoscopic approach for highly migrated intracanal disc herniations by foraminoplastic technique using rigid working channel endoscope. Spine (Phila $\mathrm{Pa}$ 1976) 2008;33:E508-15.

25. Pfirrmann CW, Oberholzer PA, Zanetti M, et al. Selective nerve root blocks for the treatment of sciatica: evaluation of injection site and effectiveness: a study with patients and cadavers. Radiology 2001;221:70411. 